\title{
Benefits of chironomid research: Perspectives from undergraduate researchers
}

\author{
Alyssa M. Anderson, Nathan J. Roberts, Tessa I. Durnin and Katherine M. Wollman \\ Department of Biology, Chemistry, Physics, and Mathematics, Northern State University, Aberdeen, South \\ Dakota, USA. \\ Corresponding Author E-mail: alyssa.m.anderson@northern.edu
}

Shifting from the research-intensive environment of graduate school to a tenure-track gig at a primarily undergraduate institution (PUI) required a bit of a change in mindset. No longer could I (Anderson) spend day after day at in the microscope or hour after hour thinking about how to analyze, interpret or present a certain data set. Instead, my time began to fill with lecture and syllabi prep and brainstorming sessions on how to best relay difficult scientific concepts to groups of naïve undergraduate students. My personal research time was suddenly shoved into tight 1-2 hour pockets of time, often at the end of the week, or restricted to summer months, when teaching obligations are limited.

While I often miss the chance to spend hours engrossed in my own research activities, I, along with many other individuals who have pursued careers at PUIs, have found ways to stay at least somewhat engaged and active in research pursuits. This happens most easily by working with and 'grooming' undergraduates who have strong potential for pursuing careers in research. Viewing research questions through the eyes of energetic and enthusiastic undergraduates who are just beginning to develop their passion for research is completely worth the tradeoff, in my mind. Helping them to develop and carry out research questions, build a passion for field and lab work, struggle through the challenges of data analysis, and confidently present their hard work (often surrounded by advanced graduate students!) at scientific conferences is incredibly rewarding, not only for them, but also for me.

Below are the 'stories' from three exceptional undergraduates I've had the honor of working with since starting my professional career at Northern State University four years ago. While these students are still exploring their ultimate career directions and may not continue their work with chironomids, I think you'll agree that it seems that they've learned an immense amount from their time spent working with midges. And, stay tuned for some future publications with their names on!

Alyssa

\section{Nathan Roberts - Environmental Science Major, graduated from Northern State University Spring 2015}

What is a Chironomid? Before my undergraduate research project, I had limited ability of how to describe this very interesting insect. During the summer of 2014 I conducted a research project involving the collection of chironomid surface floating pupal exuviae (SFPE) on the Elm and James Rivers near Aberdeen, South Dakota, USA (Fig. 1). The goal of my research was to associate chironomid diversity with the water quality of these two river systems. The land cover throughout Brown County and in particular around the Elm and James Rivers is dominated by row crops and cattle feedlots which have been shown to produce inputs detrimental to water systems. However, interestingly the Sand Lake National Wild Refuge, a 498acre area of restored grasslands which serves as habitat for migratory birds, is also located in the study area. Sand Lake is managed by the United States Fish and Wildlife Service. The James River is impounded into a reservoir at Sand Lake and the water released from the reservoir comprises the downstream flow of the James River. So, a secondary goal of my research was to examine any significant differences in chironomid diversity between areas dominated by row crops and feed lots and areas immediately downstream of the refuge.

SFPE were collected from six sites along the Elm and James Rivers monthly from June to September 2014. In addition, to correlate water conditions to chironomid diversity, water temperature and $\mathrm{pH}$ readings were collected weekly during the same time frame using a YSI Multi Parameter Tool and water clarity (turbidity) readings were also collected using a turbidity tube. SFPE were preserved in $70 \%$ ethanol, sorted, slide mounted in the laboratory and identified to genus. To date 18 samples of SFPE have been identified to genus. A total of 24 genera representing three subfamilies have been identified. Generic breakdown within 


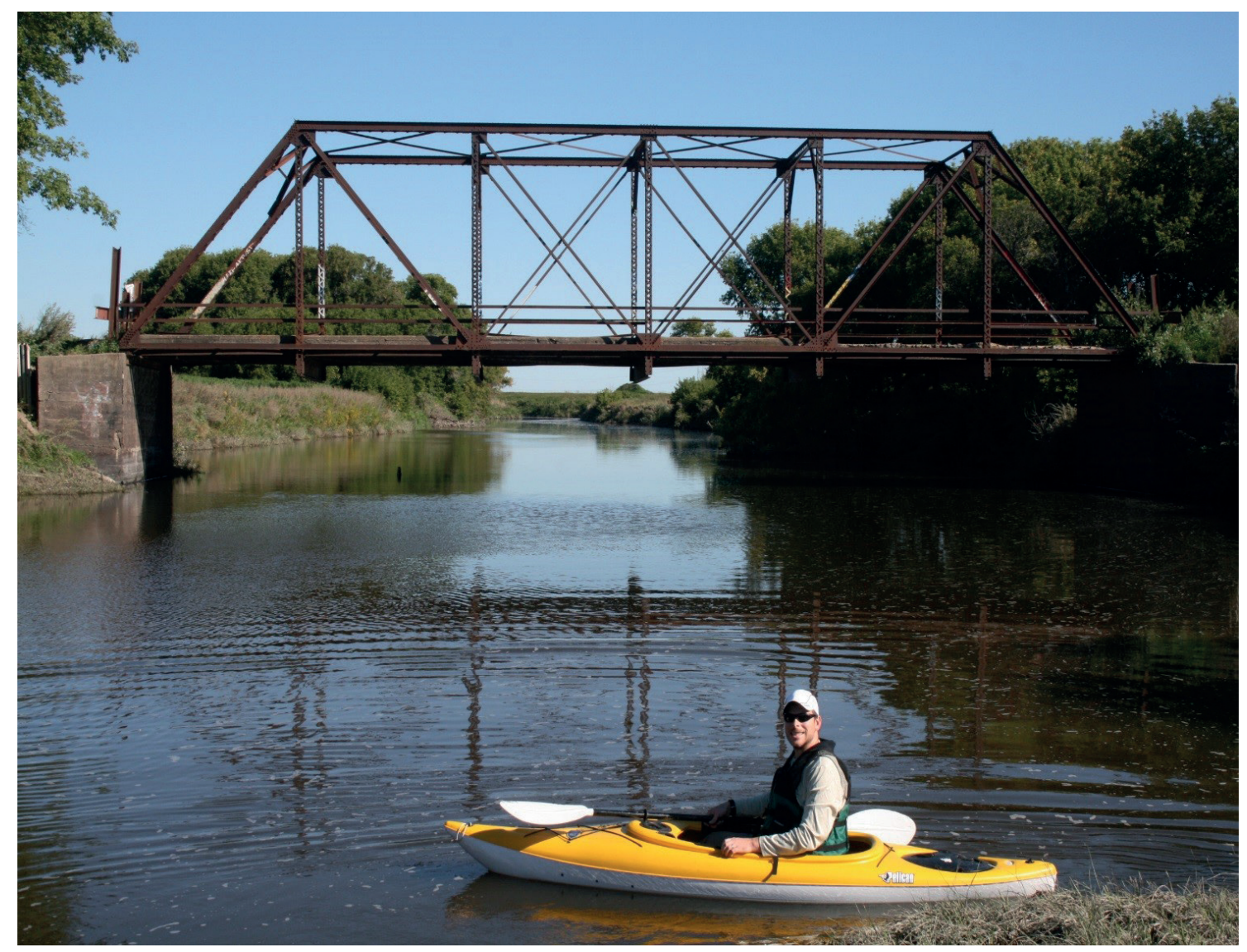

Figure 1. Nathan Roberts collecting chironomid pupal exuviae on the Elm River, South Dakota, USA, 2014.

each subfamily include 5 Tanypodinae, 3 Orthocladiinae and 16 Chironominae. This equates to a range of 11 to 18 genera present at each location.

Currently, there are 12 SFPE samples left to slide mount and identity. Also water chemistry and turbidity data still needs to be analyzed. However, I was able to present preliminary results of the project at the Society for Freshwater Science Annual Meeting in May 2015. Working with chironomids has proven to be to be a valuable experience for undergraduate research. First, working with chironomids has allowed me to appreciate that there is an abundance of biological diversity in river systems and that these organisms are very intertwined and dependent upon each other. Second, this project has taught me valuable laboratory and field skills which I hope to utilize in my future career. Third, this experience has been very helpful in my educational pursuits. Currently, I am pursing a Masters of Geographic Information Systems at Penn State University. The writing, communication, and analysis skills that I acquired through this project have been very valuable. Last, undertaking undergraduate research very much helps instill a go with the flow attitude. When I look back on my initial proposal and where my project is to this point it has changed almost 360 degrees. Also, the hypothesized results formulated at the beginning of the study appear to be very different from what we have found thus far. Therefore, I've learned to expect the unexpected. My experience working with chironomids as an undergraduate researcher has opened many doors for me and been unbelievably rewarding. Who knew all of this could be a result of something that looks like a mosquito that doesn't bite?

\section{Tessa Durnin - Biology/Environmental Science double major, graduating from Northern State Uni-} versity with a B.S. in December 2017

This past summer (2016) I was able to be a part of a stream assessment that took place roughly 75 miles east of Aberdeen, South Dakota, USA at Blue Cloud Abbey (BCA) stream. This pristine woodland stream is surrounded by areas of land that is thriving with native prairie grasses and other plants - and grazed upon by cattle downstream on a pasture as well as at the upper most region of the stream in the preserved boundaries of the abbey. My partner and I, with the help of Dr. Alyssa Anderson, completed field work over the course of a four-month period (May-August). A prescribed burn took place within a region of this sampling area in late May 2016, after our initial sampling event, and three more groups of samples were gathered monthly 


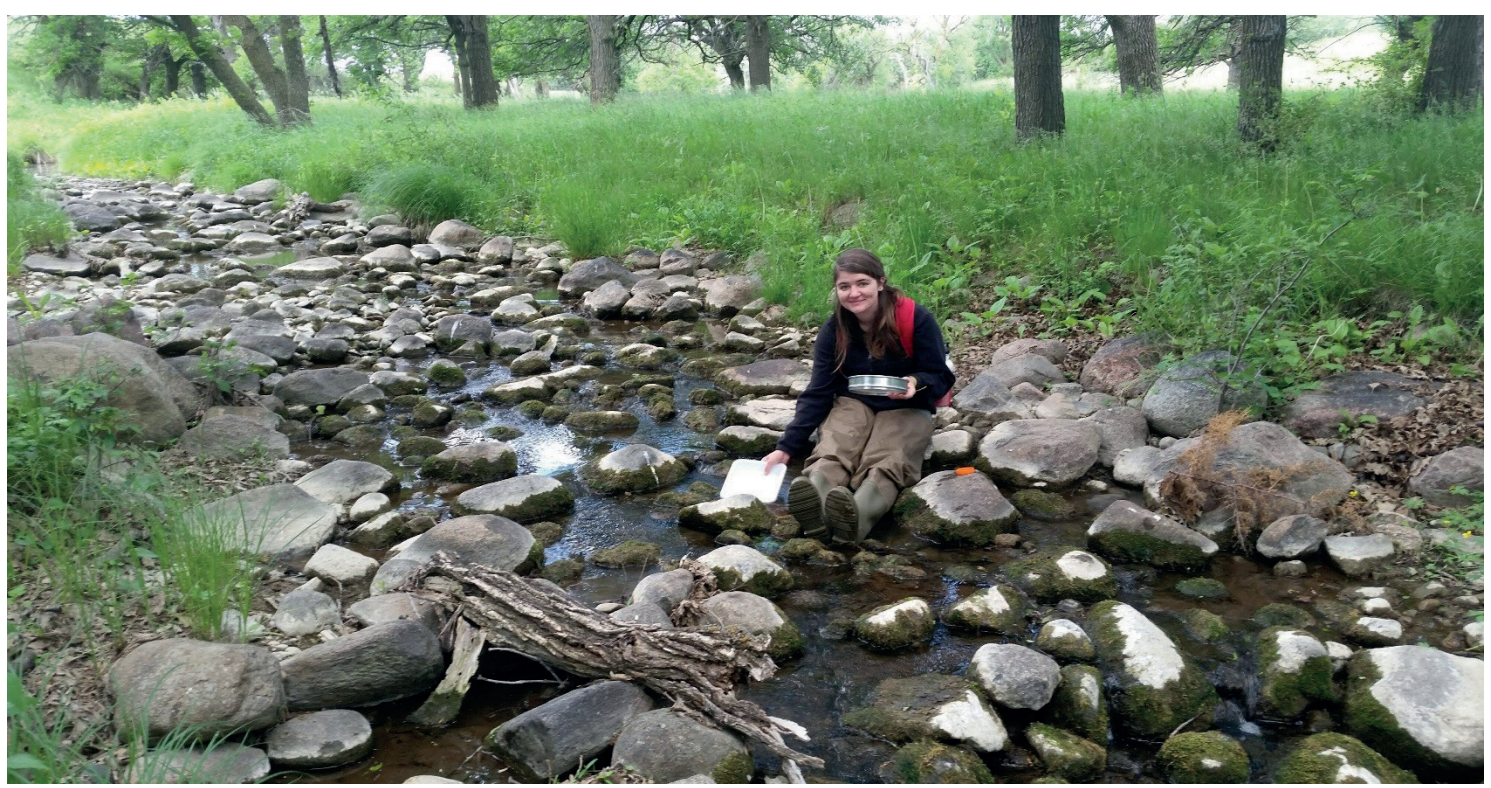

Figure 2. Tessa Durnin collecting chironomid pupal exuviae from the Blue Cloud Abbey stream, South Dakota, USA, 2016.

throughout the summer, allowing us to determine any potential consequences of the burn. Our lab work is still in progress, however completing the field experience has made me realize how much hard work and dedication is put into field work, along with how fun field work truly is.

In early May, 2016, we set up data-loggers that track temperature at four designated sites of the BCA stream. This was the hottest day of the entire experience and was also the toughest as we carried cylinder blocks (while wearing waders) and a heavy cooler filled with essential field equipment: 70\% ethanol, jars, trays, sieves, marking flags, forceps, paper, and pencils. To obtain my samples of chironomid surface floating pupal exuviae (SFPE), I would wade throughout the stream site for ten minute increments, scraping the surface of the water with a tray and straining it through a sieve (keeping in mind to look for crevices and "foamy" areas where the midge pupal exuviae may be hiding) (Fig. 2). After the ten-minute mark, I would gather a jar and ethanol, pour the sample into the jar, and place a label with the date, the site and its location, and the given time at that moment. Samples were taken back to the lab where I sorted all of the SFPE out of the initial samples, sorted the exuviae into like-groups and slide-mounted them using a dissecting microscope, and then identified them with a compound microscope and dichotomous key (slide mounting and identification is still in progress!) I've found that this process is quite difficult, time consuming, and each exuviae varies in length, color, and amount of "hairs" throughout the cephalothorax and abdomen. Dr. Anderson once told me that you basically need the patience and steady hands of a "brain surgeon" to properly get this technique down.

Even though I have yet to completely finish out this research, I have gathered extensive knowledge in field and lab work. Thanks to previous courses such as Invertebrate Zoology, Entomology, Aquatic Ecology and Watershed Management, I was able to utilize my newly acquired knowledge in the field and laboratory as an environmental research scientist. I see myself completing this research in the coming months and possibly gathering more samples this winter at BCA, as long as the stream isn't completely frozen over (one may think that insects completely "die off" in the winter, but on the contrary, certain midges thrive!).

As an undergraduate partaking in research, I look forward to my future in graduate school and am thankful for all the help I've obtained along the way. Research is basically coming up with a question that is worth finding an answer to and learning how to essentially go with the flow while making any necessary adjustments along the way. A scientist doesn't set out to prove anything, but to rather gain knowledge and to share it with others. Completing this research is one more step in helping me become a well-rounded biologist.

\section{Katherine Wollman - Biology and Environmental Science Double Major, graduated from Northern State University in December 2016}

During my time at Northern State University I participated in various research projects involving chironomids. My first experience with chironomids involved slide mounting chironomid exuviae that were 


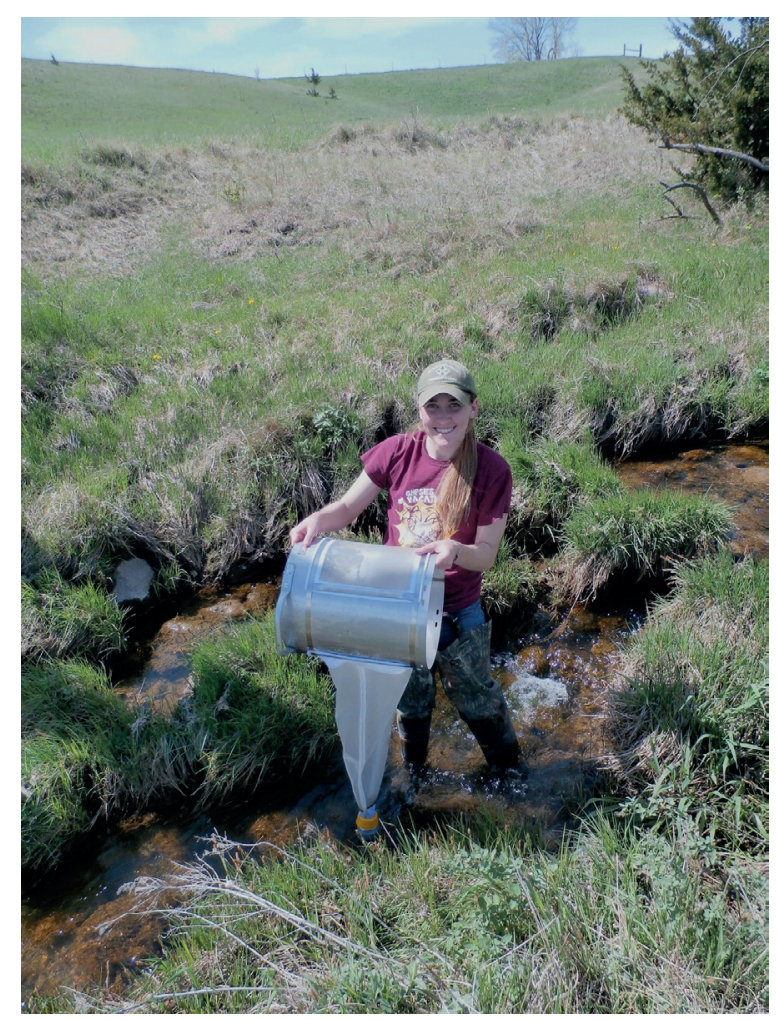

Figure 3. Katherine Wollman collecting macroinvertebrates from the Blue Cloud Abbey stream, South Dakota, USA, 2016.

the burned region, and downstream of the burn and sampled both before and several times after the burn to determine whether there were any changes to the community over the course of the sampling period (Fig. 3). Results of this work were somewhat inconclusive, as other land use factors came into play, but I did see that the overall diversity of the macroinvertebrate community declined over the course of the sampling period.

Cumulatively, these research experiences helped me learn the steps necessary to create a sound research project. Also, another important lesson I learned was how one needs to be flexible and occasionally change protocols or seek additional or alternative methods when out in the field sampling or when completing laboratory work. I was also able to gain skills in various protocols in the field and laboratory and have gained extensive experience in identification. Finally, learning how to thoroughly analyze my results broadened my understanding of factors affecting stream diversity, which made the end results of my work more rewarding.

Each of these research projects helped to solidify my decision to continue my studies in graduate school; without these experiences, I would not have even considered graduate education. Ultimately, these projects made my undergraduate experience feel more valuable because I was able to take charge of a project and do things on my own and apply skills learned about in lecture in the field and lab. Research gives a you a challenge and makes you stretch your mind.

\section{References}

Wollman, K.M., Kranzfelder, P. and Anderson, A.M. 2016. Chironomidae (Insecta: Diptera) of San Salvador, Bahamas: A search for new species. - Proceedings of the South Dakota Academy of Science 95: 143.

Article submitted 15. December 2016, accepted by Torbjørn Ekrem 16. December 2016, published 19. December 2016. 